\title{
The influence of gene Rv3671c in Mycobacterium bovis to its replication and acid resistance

\author{
Weidong Lin ${ }^{*}, \mathrm{a}, \mathrm{b}$, Ting Xin ${ }^{*}, \#, \mathrm{a}$
}

${ }^{a}$ Institute of Animal Sciences (IAS), Chinese Academy of Agricultural Sciences (CAAS), Beijing, China

${ }^{b}$ Molecular and Cellular Biology, Gembloux Agro-Bio Tech University of Liège (ULg), Gembloux, Belgium

* Weidong Lin and Ting Xin contributed equally to this work as co-first authors.

${ }^{*}$ Corresponding authors:

Institute of Animal Sciences, Chinese Academy of Agricultural Sciences,

2 Yuanmingyuan South Street, Haidian District,

100193 Beijing, China 


\begin{abstract}
To study the effect of Marp protein in Mycobacterium bovis to the acid resistance and growth performance, this research constructed a knockout mutant ( $\triangle M a r p$ ) with mycobacteriophage, complemented strain ( $\triangle$ MarpComp), and overexpressing strain (PmvRv3671) of the gene Rv3671c with pmv261 plasmid. Culturing them in standard 7H9 medium to the early logarithmic phase and transferring them into $7 \mathrm{H} 9$ medium at $\mathrm{pH} 6.6$ and pH 5.0 and maintenance solution at $\mathrm{pH} 6.6$ and $\mathrm{pH}$ 4.5. Likewise, macrophages Raw 264.7 were infected with multiple infections at 10 . The results showed that while they grew in $7 \mathrm{H} 9$ medium at $\mathrm{pH} 5.0$ or $\mathrm{pH} 6.6$ and maintenance buffer at $\mathrm{pH} 4.5$, the lived number of over-expressing strain PmvRv3671 is more than wild-type strain M.bovis, $\Delta$ MarpComp and $\Delta$ Marp on the $14^{\text {th }}$ day. After removing the effects of citrate solution, it can be found that the acid resistance abilities of them all are significantly lower on the $14^{\text {th }}$ day than that on the $5^{\text {th }}$ day. Using them infected Raw264.7 macrophages with IFN $\gamma$ stimulation, the growth rate of the PmvRv3671 is better than An5, $\Delta$ Marp and $\Delta$ MarpComp. In conclusion, Rv3671c over-expressing strain had shown a better growth ability than wild-type An5, $\Delta$ Marp and $\Delta$ MarpComp under acidic environment. When exposed to $\mathrm{pH} 4.5$ citrate maintenance solution for a long time, acid resistance abilities of them all have become weaker.
\end{abstract}

Keywords: Mycobacterium bovis; Acid resistance; Marp; Serine proteinase; Raw264.7.

\title{
1 Introduction
}

Tuberculosis is an important infectious disease with large number of deaths caused by a single pathogen in the world and is also considered the leading cause of death after AIDS (WHO, 2019).

The main pathogenic bacteria of tuberculosis are Mycobacterium tuberculosis (Mtb) and Mycobacterium bovis (M. bovis). Mechnikov indicated that macrophages would kill most of the ingested microorganisms by acidifying its interior, but Mtb could use its waxy cell wall to resist this acidification (Mechnikov, 1988). According to previous reports, when Bacille Calmette-Guérin (BCG), an attenuated variant of $M$. bovis, was exposed to $\mathrm{pH} 5.5$ in vitro, it ceased replicating and Mtb continued to divide slowly unless the $\mathrm{pH}$ was lowered to 4.5 . With the addition of $0.5 \mathrm{mM}$ nitrite to 7H9 medium at pH 5.5, Mtb would also stop replicating. However, it is in a balanced state, where the Mtb could survive for several days, and the bacterial can return to a normal growth rate after replacing the medium with standard 7H9 (Bryk et al., 2008; Gold et al., 2012; Vandal et al., 2008). Based on that, Vandal used the acidic environment to screen 10,100 MTB transposon mutants and found a mutant that cannot maintain its intracellular pH homeostasis under a pH 4.5 environment (Vandal et al., 2008). Because the gene $R v 3671 c$ product played an essential role in the acid resistance of Mtb, it is named as Mycobacterial acid resistance protease (Marp). Marp was identified as a transmembrane serine peptidase with a protease domain located in the periplasm, as confirmed by a prudent analysis of its mutations, homology modelling, crystallography, and substrate profiling (Biswas et al., 2010; Small et al., 2013; Vandal et al., 2008).

We had confirmed that Marp was also presented in M. bovis (data not shown), but it remains as speculation if the role of this gene in $M$. bovis is related to acid resistance or if there are other biological functions involved in, such as the growth. Furthermore, because $M$. bovis had more extensive hosts beyond human susceptibility, it could also infect domestic animals and wild animals under natural circumstances (Pesciaroli et al., 2014). Thus further studies on M. bovis are extremely meaningful to public safety (Gao et al., 2019; Xin et al., 2018).

In order to study the functions and biological characteristics of the gene Rv3671c in M. bovis, this research aimed to construct a knockout mutant ( $\triangle M a r p)$, complemented strain ( $\triangle$ MarpComp), and overexpressing strain (PmvRv3671) of the gene Rv3671c. After that, they were cultured in standard or acidic mediums and used to infect macrophages to closely examine and compare their difference. Subsequently, it was possible to state the functions and influences of gene Rv3671c in M. bovis to its growth and acid resistance. 


\section{Materials and methods}

\subsection{Analyzing Rv3671c sequence and designing the primers}

According to the genome sequence of M. bovis (AWPL01000069.1) registered in GenBank, the search for the $R v 3671 c$ sequence showed that there is a high homology sequence between 158384 and 159586, consistent with the characteristics of Rv3671c (CP003248.2) in MTB H37Rv. As shown in Figure 1, the red band of the $R v 3671 c$ gene was knocked out of $M$. bovis. The blue bands of the upstream and downstream fragments of the gene are called the left arm and the right arm individually. The primers LFP / LRP and RFP / RRP are used to amplify the left and right arms. The primers LRP and RFP need to contain the partial sequence of the left and right ends of the gene to be eliminated. In this case, the verification primer (LYZ / RYZ) is used in the subsequent PCR verification stage, and the verification primer is designed upstream of the LFP and downstream of the RRP (indicated by the black band). The primer sequences are all listed in Table 1.

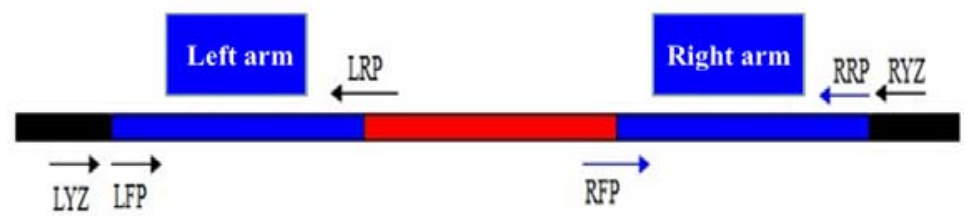

Figure 1. Schematic diagram design of primers. The blue bands of the upstream and downstream fragments of the gene are called the left arm and the right arm individually. LFP / LRP and RFP / RRP are used to amplify the left and right arms. The primers LRP and RFP need to contain the partial sequence of the left and right ends of the gene to be knocked out. The verification primer (LYZ / RYZ) is used in the subsequent PCR verification stage, and the verification primer is designed upstream of the LFP and downstream of the RRP (indicated by the black band).

Table 1. Primers used in this study.

\begin{tabular}{cl}
\hline $\begin{array}{c}\text { Primers } \\
\text { name }\end{array}$ & Sequence $\left(5^{\prime} \rightarrow 3^{\prime}\right)$ \\
\hline Marp $\mathrm{F}$ & GGAATTCATGACCCCGTCGCAGTGGCTGGATATCGC \\
Marp $\mathrm{R}$ & CAAGCTTGTCAGCTGACGCAGGCCCCGGT \\
Pmv261-F & GTTGTCGTTGGCGGTCAT \\
Pmv261-R & ATGATGGCCGGACAAACAACAGATAA \\
LFP & TTTTTTTTGCCTAAATGGCCCCTGCAAGCGGGAGCGACC \\
LRP & TTTTTTTTGGCCTTTCTGGCCCAGCATTGAGCCCAGCGCA \\
RFP & TTTTTTTCCATAGATTGGCAGGTGCTCGGTGGGTTTC \\
RRP & TTTTTTTCCATCTTTTGGCGAGGCCGCTGGTCATACTG \\
LYZ & GTTAGCGGCTTGAAGAGGA \\
RYZ & AACGGCATCCGATTCCAC \\
\hline
\end{tabular}

\subsection{Construction of M. bovis Rv3671c knockout mutants}

For this chunk of the experiment, native Rv3671c was replaced in An5 M. bovis with a hygromycin-resistant cassette via allelic exchange following the protocol as reference (Bardarov et al., 2002). The DNA segments flanking upstream and downstream regions of the Rv3671c gene (named left arm and right arm) were amplified by PCR using suitable primers (as shown in Figure 2) and digested 
by SrfI and PflMI separately. The allelic exchange substrate was then cloned into the suicidal vector p0004S directionally, on either side of the hygromycin resistance-sacB gene cassette $(3.6 \mathrm{~Kb})$. The recombinant vector p0004S was also linearized by PacI digestion, and packaged into the temperature-sensitive TM4 shuttle phasmid phAE159 with digesting by PacI digestion for generating the specialized transducing mycobacteriophage. The phage could be amplified in $M$. smegmatis $\mathrm{mc}^{2} 155$, and high-titer phage particles were then prepared at a replication permissive temperature of 30 ${ }^{\circ} \mathrm{C}$. Further on, the gene was deleted from M. bovis An5 by specialized transduction. Allelic exchange occurred as a result of a double crossover between the homologous DNA arms flanking the disrupted gene. Transductants were then screened for gene disruption by PCR using a forward primer for left arm (LFP) and right arm reverse primer (RRP). Gene deletion could be confirmed by western blotting using monoantibody against Marp. The Rv3671c gene disruption strain was designated $\Delta$ Marp.

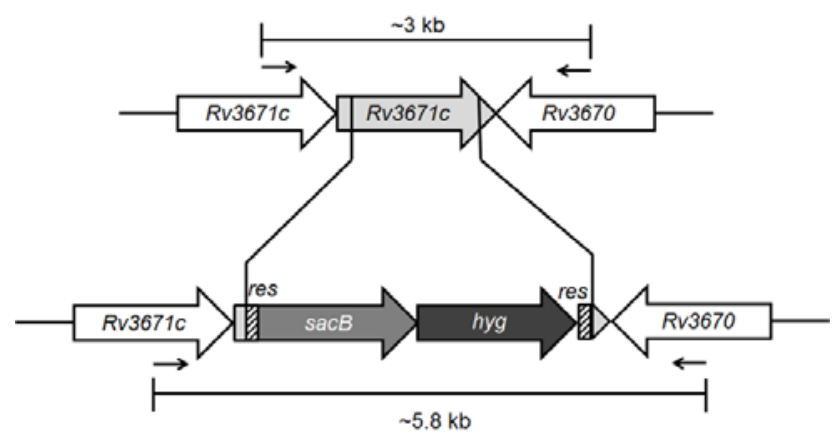

Figure 2. Schematic diagram of allelic replacement of the Rv3671c gene. The constructed phagemid contains the DNA segments flanking upstream and downstream regions of the Rv3671c gene (named left arm and right arm). So when the packaged phage infected with $M$. bovis, native Rv3671c was replaced in $M$. bovis An5 with a hygromycin-resistant cassette via allelic exchange.

\subsection{Construction of complemented strain and overexpressing strain}

For completing the $\triangle M a r p$ and preparing an overexpressing strain, the $R v 3671 c$ gene was amplified from $M$. bovis genomic DNA by PCR. After that, it was cloned into the E. coli-Mycobacterium shuttle plasmid pMV261 with the primer-incorporated Hind//I and EcoR I sites to yield the recombinant plasmid named pMV261-Marp. This recombinant plasmid was then electroporated into the mutant strain $\triangle$ Marp and wild-type strain An5 with $2.5 \mathrm{kV}, 1,000 \mathrm{ohms}$ and $25 \mu \mathrm{F}$. Electroporation was performed as previously described (Snapper et al., 1990). The complemented colonies were selected from plates containing hygromycin and kanamycin, and overexpressing strains were selected from plates containing kanamycin. PCR and western blot were used to identify the genome and proteins of selected $\Delta$ MarpComp and PmvRv3671.

\subsection{Media and Culture Conditions.}

M. bovis was cultured at $37{ }^{\circ} \mathrm{C}$ on Middlebrook $7 \mathrm{H} 10$ agar containing $10 \%$ oleic acid-albumin-dextrose-catalase (OADC) and $0.5 \%$ glycerol or in $7 \mathrm{H} 9$ standard medium (Middlebrook 7H9 broth supplemented with $0.2 \%$ glycerol, $10 \%$ OADC, and $0.05 \%$ Tween 80 or $0.02 \%$ Tyloxapol). The standard $7 \mathrm{H} 9$ was acidified to $\mathrm{pH} 4.5$ or $\mathrm{pH} 5.0$ with $2 \mathrm{~N} \mathrm{HCl}(M$. bovis couldn't grow in $7 \mathrm{H} 9$ at $\mathrm{pH} 4.5$ ). According to the previous report, at least two components of the 7H9 medium can become toxic to MTB at $\mathrm{pH}$ 4.5: one associated with Tween and another associated with albumin, and both may release free fatty acids at low pH (Vandal et al., 2008). So the experiment needed to be supplemented with culturing these bacteria on $7 \mathrm{H} 9$ at $\mathrm{pH} 5.0$. Phosphate-citrate (pcit) buffer at $\mathrm{pH} 4.5$ or $\mathrm{pH} 6.6$ was made from $200 \mathrm{mM}$ sodium phosphate and $100 \mathrm{mM}$ citric acid. Pcit buffer at $\mathrm{pH} 4.5$ or $\mathrm{pH} 6.6$ 
contained $0.02 \%$ tyloxapol (pcit-6.6 buffer or pcit-4.5 buffer). Strains with antibiotic resistance cassettes growled in the presence of $75 \mu \mathrm{g} / \mathrm{mL}$ hygromycin B (4Marp), $20 \mu \mathrm{g} / \mathrm{mL}$ kanamycin (PmvRv3671), or a combination of $75 \mu \mathrm{g} / \mathrm{mL}$ hygromycin B and $20 \mu \mathrm{g} / \mathrm{mL}$ kanamycin (COMP) (van Kessel and Hatfull, 2007).

\subsection{Measurement of acid resistance and growth characters}

Colony-forming units (CFU) were determined by plating serial dilutions of the suspensions on 7H10 agar plates. The early-log-phase (within 3days) cultures were washed with 7H9- pH6.6, 7H9- pH5.0, 7H9- pH4.5, pcit-ty-6.6 or pcit-ty- 4.5 medium and centrifuged at $120 \mathrm{~g}$ for $10 \mathrm{~min}$. Also, the single-cell suspensions were adjusted to $\sim 5 \times 10^{6} \mathrm{CFU} / \mathrm{mL}\left(\sim \mathrm{OD}_{600}=0.015\right)$ in $7 \mathrm{H} 9-\mathrm{pH} 6.6,7 \mathrm{H} 9-\mathrm{pH} 4.5$, pcit-ty- 6.6 or pcit-ty- 4.5 medium and incubated at $37{ }^{\circ} \mathrm{C}$. The $\mathrm{pH}$ and $\mathrm{OD}_{600}$ values were also recorded for further evaluation.

\subsection{Intracellular viability of the M. bovis}

The intracellular viability of $\triangle$ Marp was determined using macrophage infection RAW264.7 cells, cultured in DMEM medium supplemented with $10 \%$ fetal bovine serum (FBS) and 10 mM HEPES. The cells were then seeded onto 24 well plates $\left(10^{6}\right.$ cells/well $)$ with or without $10 \mathrm{ng} / \mathrm{mL}$ murine IFN- $\gamma$ (R\&D Systems). Sixteen hours later, macrophages were used to infect in triplicates with wild type AN5, $\Delta$ Marp, PmvRv3671, and the $\Delta$ MarpComp at a multiplicity of infection of 0.1 and then washed with PBS 4 hours later to remove the non-phagocytosed bacilli. The infected macrophages were then incubated with fresh DMEM supplemented with $2 \% \mathrm{FBS}$ at $37^{\circ} \mathrm{C}$ in the presence of $5 \% \mathrm{CO} 2$ and it was necessary to replace the medium every $48 \mathrm{~h}$. Intracellular bacilli could be recovered by lysing infected macrophages with $0.5 \%$ Triton X-100 and enumerated bacteria by plating serial dilutions of the lysate on $7 \mathrm{H} 10$ or $7 \mathrm{H} 11$ agar plates in 48,96 , and 144hours post-infection.

\subsection{Statistical analysis}

GraphPad Prism 5 software (GraphPad Software, Inc., USA) or SPSS software version 20.0 (IBM, Inc., USA) was used for the data analysis and all the data expressed as the mean \pm standard error of the mean. Differences in the survival rates and growth ratios, between the different groups (An5, $\Delta$ Marp , $\triangle$ MarpComp, and PmvRv3671) were determined by two-way ANOVA and considered statistically significant at $p<0.05$ or highly significant at $p<0.01$.

\section{Results}

\subsection{Amplifying the upstream and downstream homology arms of the Rv3671c gene}

The amplification results of the upstream and downstream homology arms of the target gene by PCR are displayed in Figure 3a, and the sizes of PCR amplification products are consistent with the expected length. The upstream and downstream of homologous arms were digested by restriction enzymes SrfI and PflMI, respectively, and the digested products were recovered. After connecting the upstream and downstream to the vector p0004s, named p0004S-4Marp, it was transformed into E.coli DH5a. After culturing them on LB agar with hygromycin B, clones were picked up, and their plasmids were identified by PCR. The P0004S-AMarp plasmid was then packaging into phAE159 and transformed into HB101 competent and finally the plasmid was extracted from positive colonies by digestion identification (the results are shown in Figure $3 b$ ). 

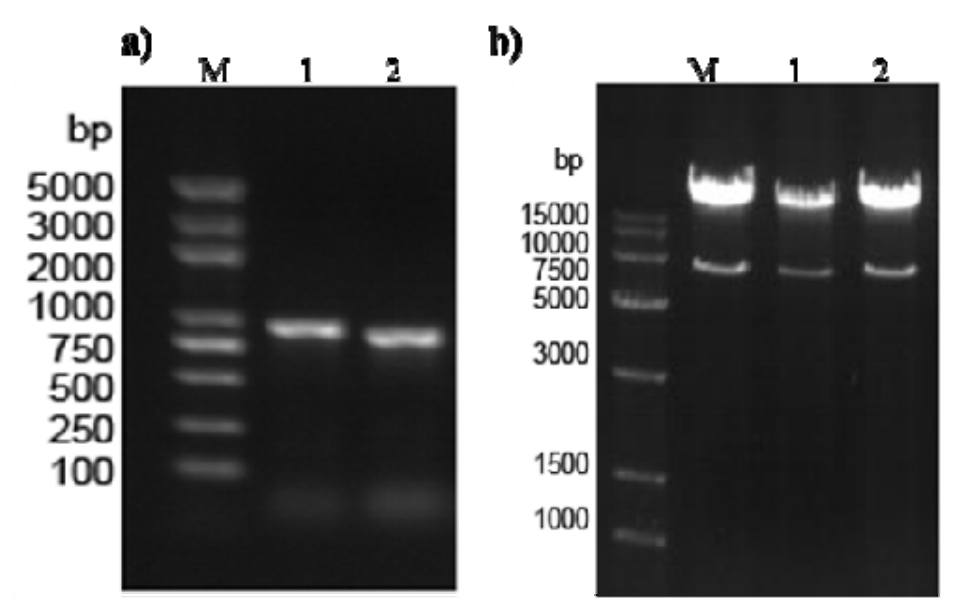

Figure 3. The process to build recombinant phAE159. a): PCR amplification results of the upstream and downstream homology arms of the Rv3671c gene. Extracting the DNA from M. bovis AN5, the template was used to amplify the upstream (lane 1) and downstream homology arms (lane 2) of the Rv3671c gene.

The results were analyzed by agarose gel electrophoresis. b): The digestion results of recombinant

phAE159 with SrfI and PfIMI. We selected 3 colonies of recombined HB101 from LB agar with hygromycin B. After obtaining their recombinant phAE159-AMarp, the phagemid was digested by SrfI and PflMI and analyzed by agarose gel electrophoresis (lane1-3).

\subsection{Preparation of high-titer phage lysate}

Recombinant phagemid was transfected into Mycobacterium smegmatis $\mathrm{mc}^{2} 155$ competence by electroporation, and the plaques (shown in Figure 4a) were picked up to prepare high-titer phage lysate. The phage was amplified in M. smegmatis $\mathrm{mc}^{2} 155$ and infected $M$. bovis for homologous recombination. The colonies of $M$. bovis were selected and detected by primers of LYZ and RYZ. As a final stage, the PCR results were closely examined by agarose gel electrophoresis as Figure $4 \mathrm{~b}$ and showed that the hygromycin resistance gene successfully replaced the Rv3671c in $\Delta$ Marp mutant, and the results of western blotting (Figure 4c) proved that there was no Marp expression in $\Delta$ Marp mutant, indicating that $\Delta$ Marp strain was successfully constructed.

a)

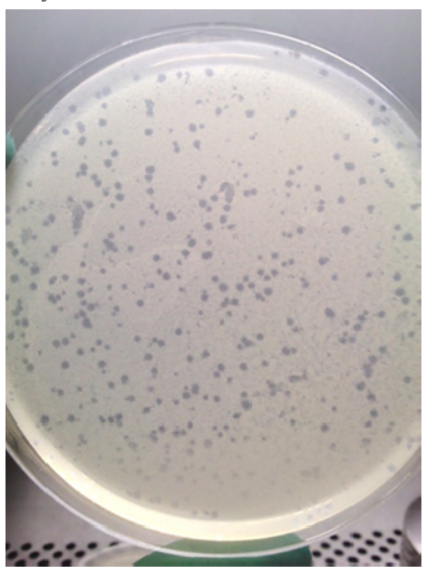

b)

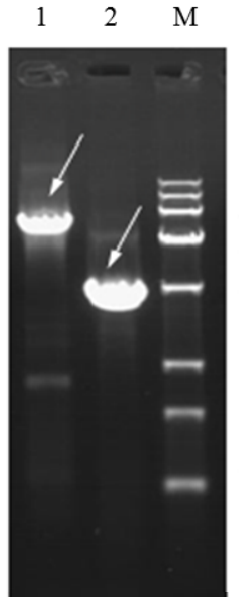

c)

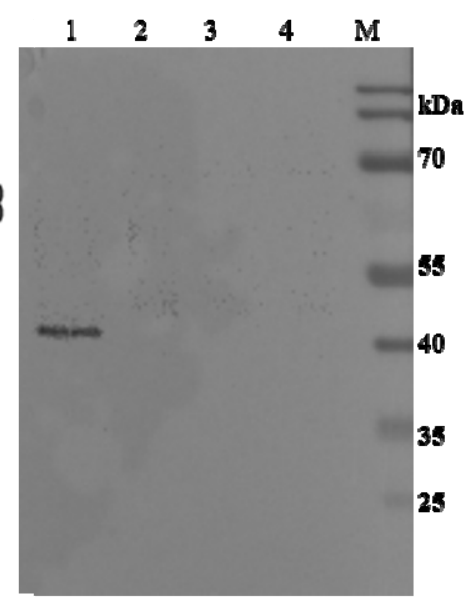

Figure 4. Identification of $\Delta$ Marp mutant. a): Plaque formed by the constructed bacteriophage. Recombinant phagemid was transfected into $M$. smegmatis $\mathrm{mc}^{2} 155$ competence by electroporation, and 
the plaques will show up like above. b): Identification results of $\triangle$ Marp mutant by PCR. The colonies of $M$. bovis and wild-type AN5 were selected and detected by primers of LYZ and RYZ. The white arrow indicates the target DNA fragment. Lane 1 showed the DNA ladder that $R v 3671 c$ was replaced with $s a c B$ gene cassette in the mutant clone; lane 2 showed the original target $R v 3671 c$ gene DNA fragment in wild-type AN5. It means that the hygromycin resistance gene successfully replaced the $R v 3671 c$ in $\Delta$ Marp mutant. c): Identification of $\Delta$ Marp mutant by western blot. We selected 3 colonies of recombineering colonies from 7H10 agar with hygromycin B and extracted their total proteins.

Using the Marp monoantibody, the Marp were detected in these colonies by western blot with the total proteins of wild-type An5 as a positive control. The results showed that Marp can be detected from wild-type AN5 (lane1) but there was no Marp expression in $\triangle$ Marp mutant (lane2-4).

\subsection{Construction of Complemented Strain and}

\section{overexpressing strain}

PCR amplification helped to obtain the PCR products Rv3671c; PMV261 plasmids were digested with EcoRI and Hind III at the same time. After that, their digested products were ligated together, and then the conjunction was transformed into E.coli DH5a competent cells; the bacteria needed to be cultured on LB agar with kanamycin to select colonies whose plasmids were used to identify by PCR. The recommend plasmids were extracted and electroporated individually into the $\Delta$ Marp and An5; they were then cultured on $7 \mathrm{H} 10$ agar at $37^{\circ} \mathrm{C}$ for 4 weeks. The identification results about the selected colonies were obtained by PCR shown in Figure 5.

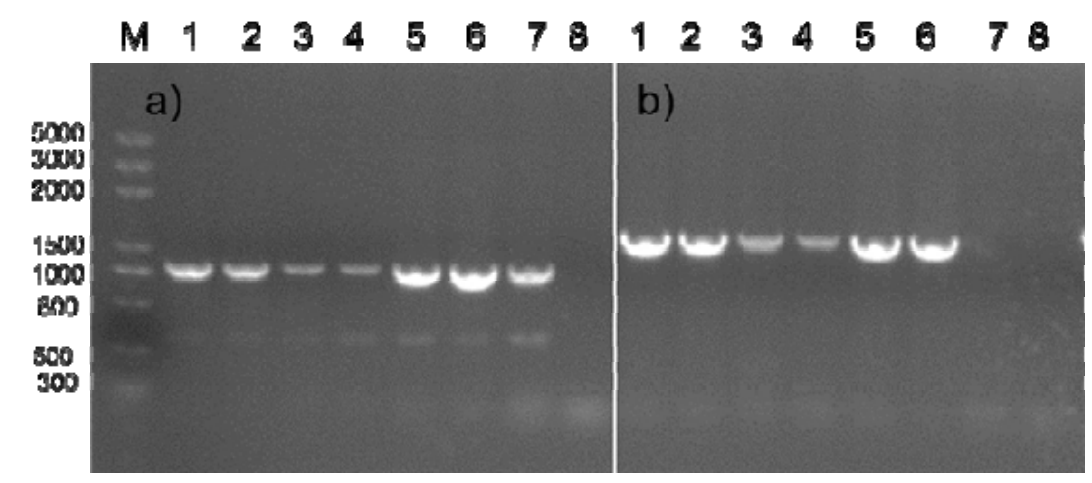

Figure 5. Identification results of $\triangle \mathrm{MarpComp}$ and PmvRv3671 by PCR. The recombined bacteria ( $\triangle$ MarpComp and PmvRv3671) were selected, and their DNA was extracted. Their DNA was detected by PCR with primers Marp (Figure 5a) or Pmv261 (Figure 5b); meanwhile, the An5 genome was used as the positive control, and $\mathrm{ddH}_{2} \mathrm{O}$ was used as the negative control. a) The PCR amplification results with

Marp primers. It showed that the $R v 3671 c$ gene could be found in $\Delta$ MarpComp strains (lane 1-3),

PmvRv3671 strains (lane 4-6) and An5(lane 7), and could not be found in the negative control (lane 8); it means that the PCR process was nonpolluted; b) The PCR amplification results with PMV261 primers, showing that Pmv261-Marp plasmid could be found in $\Delta$ MarpComp strains (lane 1-3) and PmvRv3671 strains (lane 4-6), and it could not be seen in An5 (lane 7) and $\mathrm{ddH}_{2} \mathrm{O}$ (lane 8).

\subsection{Measurement of acid resistance and growth characters}

All of the four types of bacteria were cultured in 7H9-pH6.6 and 7H9-pH5.0 mediums at $37^{\circ} \mathrm{C}$ (they all cannot grow at 7H9-pH 4.5), and their growth curves were measured during 14 days. The growth results of $\mathrm{OD}_{600}$ value and $\mathrm{CFU}$ about the four types of bacteria, in standard 7H9-pH6.6 medium, within 14 days are shown as Figures $6 \mathrm{a}$ and $6 \mathrm{~b}$. Figure $6 \mathrm{a}$ showed that the wild-type strain An5 presented the largest increase in $\mathrm{OD}_{600}$ values over time, and the other three strains were similar. Figure $6 \mathrm{~b}$ represents the relationship between the number of living bacteria and the change of time. As it can 
be seen, the PmvRv3671 has more CFU than the other three. To sum up, the number of living bacteria of PmvRv3671 is $10^{8.55}$ on the $14^{\text {th }}$ day, 5 times more than that of $\Delta$ Marp. It suggested that the deletion of gene $R v 3671 c$ might weaken the growth ability of the strain in a neutral environment.

The growth curve of $\mathrm{OD}_{600}$ value and $\mathrm{CFU}$ of the four types of bacteria, in $7 \mathrm{H} 9$ at $\mathrm{pH} 5.0$ medium, within 14 days are exhibited in Figures $6 \mathrm{c}$ and $14 \mathrm{~d}$. Figure 6c shows that the $\mathrm{OD}_{600}$ value of $\Delta$ Marp on the $14^{\text {th }}$ day was 0.30 , the lowest value among these four strains. The $\mathrm{OD}_{600}$ value of An5, $\triangle$ MarpComp, and PmvRv3671 were 1.17, 0.98, and 1.32, respectively. From the comparison of the count of living bacteria in Figure 6d, it can be seen that the PmvRv3671 had the highest value of $\log _{10} \mathrm{CFU} / \mathrm{mL}-8.95$ in $7 \mathrm{H} 9-\mathrm{pH} 5.0$, almost $0.4 \operatorname{logs}$ more than that in 7H9-pH6.6. The $\log _{10} \mathrm{CFU} / \mathrm{mL}$ value of $\triangle$ marpComp and An5 are similar - 8.16 and 8.01 - either of them is more $2.0 \mathrm{log}$ than the value 6.68 of $\triangle M a r p$. This indicates that the Rv3671c gene affected the growth of Mycobacterium bovis in 7H9-pH 5.0 medium.

In summary, PmvRv3671 had more living bacteria in an acidic environment than a neutral environment. Moreover, the $\triangle$ Marp cannot maintain the ability to grow in an acidic environment like wild-type strain, so it suggested that, due to the existence of gene $R v 3671 c$, $M$. bovis could grow in an acidic environment like in the neural environment. In other words, $R v 3671 c$ may participate in the acid-resistant process of $M$. bovis and the process of maintaining the intracellular neutral environment under acidic environment.
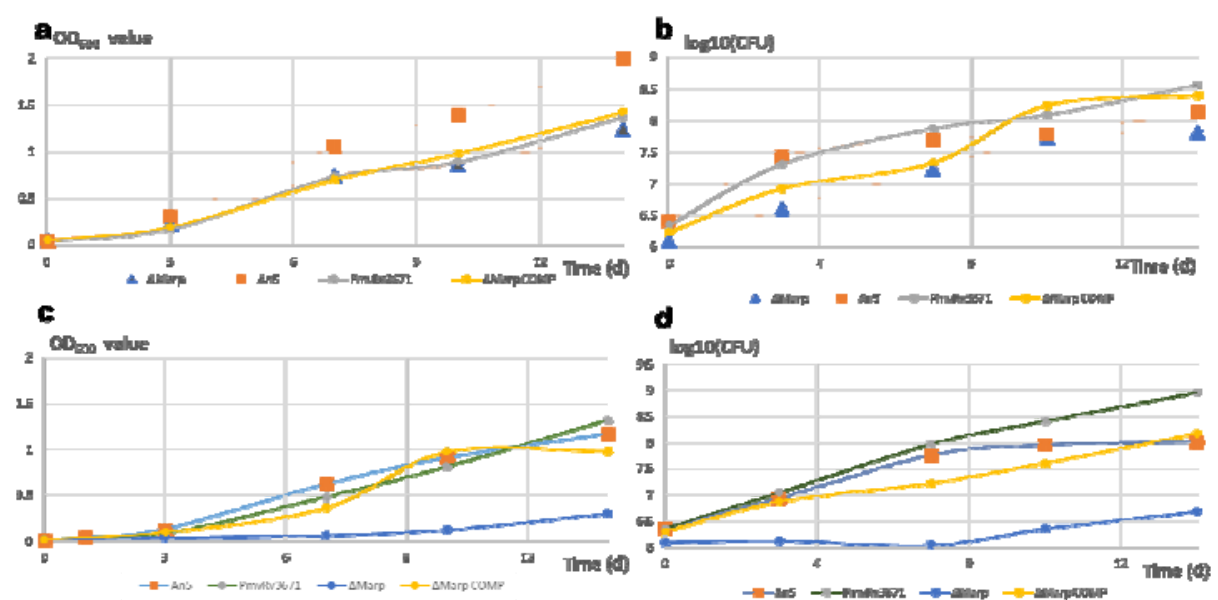

Figure 6. Growth results of An5, $\triangle$ Marp, $\triangle$ MarpComp, and PmvRv3671 respectively at pH 5.0 and pH $6.67 \mathrm{H} 9$. All of the four types of bacteria were cultured in $7 \mathrm{H} 9-\mathrm{pH} 6.6$ and $7 \mathrm{H} 9-\mathrm{pH} 5.0$ medium at $37^{\circ} \mathrm{C}$, and their growth curves were measured during 14 days. (a) $\mathrm{OD}_{600}$ value of An5, $\triangle$ Marp, $\triangle$ MarpComp, and PmvRv3671 at pH 6.6 in 7H9; (b) Quantification of CFU of An5, $\Delta$ Marp, $\triangle$ MarpComp, and PmvRv3671 at pH 6.6 in 7H9; (c) $\mathrm{OD}_{600}$ value of An5, $\Delta$ Marp, $\Delta$ MarpComp, and PmvRv3671 at pH 5.0 in 7H9; (d) Quantification of CFU of An5, AMarp, $\triangle$ MarpComp, and PmvRv3671 at pH 5.0 in $7 \mathrm{H} 9$. Bacterial input was $0.5 \times 10^{7} \mathrm{CFU} \mathrm{ml}^{-1}$.

\subsection{Determining the different acid resistance of $\Delta$ Marp,} $\Delta$ MarpComp, PmvRv3671, and An5

For this step, the four strains were cultured in a biochemical incubator at $37^{\circ} \mathrm{C}$ at $\mathrm{pH} 4.5$ and $\mathrm{pH} 6.6$ in pcit-ty maintenance buffer. We have measured their survival rates for 14 days. As can be seen from Table 2, on the $5^{\text {th }}$ day, the survival rates of $\Delta$ Marp, $\Delta$ MarpComp, An5, and PmvRv3671 in the acidic (pH4.5) environment were 12.94\%, 25.65\%, 21.02\%, and 109.4\%, respectively; meanwhile, the survival rate of PmvRv3671 in the acidic environment was even better than that in a neutral environment.

Only after dividing the survival rate of each strain at $\mathrm{pH} 4.5$ by their own survival rate of $\mathrm{pH} 6.6$ in 
pcit buffer, the corrected survival rate could be calculated. It can be used to evaluate the resistance to the pressure from the acid environment without the effect of pcit buffer. The adjusted values revealed that the survival rate of the PmvRv3671 (161.4\%) in pH4.5 environment was very significantly higher than that of the $\triangle$ MarpComp $(30.8 \%)$ and An5 $(28.8 \%)$, both significantly higher than $\Delta$ Marp $(18.56 \%)$.

According to statistic analysis results showed in Table 2, on the $14^{\text {th }}$ day, the survival rate of $\Delta$ Marp in the pcit-6.6 is $69.97 \%$, higher than that of the other three strains. Next is the $\Delta$ MarpComp $(12.67 \%)$, more inflated than the An5 (6.03\%) and PmvRv3671 (5.11\%) in the neutral environment. Contrarily, the survival rate of PmvRv3671 in the pcit-4.5 is 1.7\%, also higher than that of $\Delta$ MarpComp $(0.72 \%)$, An5 $(0.68 \%)$ and $\triangle$ Marp $(0.09 \%)$. The survival rates of $\Delta$ Marp $(0.09 \%)$ is the lowest one among these four strains on $14^{\text {th }}$ day. Without the influence of the maintenance solution, the survival rate of PmvRv3671 (33.29\%) is still more advantageous than An5 (11.26\%), $\Delta$ MarpComp (5.66\%) and $\Delta$ Marp $(0.13 \%)$.

In summary, we found that the survival rate of the PmvRv3671 is the lowest one in pcit-6.6 and the highest one in pcit-4.5 among the four strains. On the other hand, $\Delta$ Marp is particularly stable in the environment of pcit-6.6, even on the $14^{\text {th }}$ day, the survival rate was the same as the $5^{\text {th }}$ day $(69.97 \%)$. Besides, comparing the corrected data of the four strains, the acid resistance capacity of each four strains on the $14^{\text {th }}$ day has decreased significantly than that on the $5^{\text {th }}$ day. These indicated that the presence or absence of the $R v 3671 \mathrm{c}$ gene made a notable influence on the survival rate of M. bovis in a neutral and acidic environment, and $\triangle$ Marp remained part of acid resistance, but the ability of $\Delta$ Marp also became weaker with the time extended.

Table 2. Survival rates of $M$. bovis An5, $\Delta$ Marp, $\Delta$ MarpComp, and PmvRv3671 respectively at $\mathrm{pH}$ 4.5 and $\mathrm{pH} 6.6$ maintenance solutions (pcit buffer ) .

\begin{tabular}{|c|c|c|c|c|c|c|}
\hline & \multicolumn{3}{|c|}{$5 \mathrm{~d}$} & \multicolumn{3}{|c|}{$14 d$} \\
\hline & pcit- 6.6 & pcit-4.5 & $\begin{array}{c}\text { pcit-4.5/pcit-6.6 } \\
\#\end{array}$ & pcit- 6.6 & pcit-4.5 & $\begin{array}{c}\text { pcit-4.5/pcit-6. } \\
6^{\#}\end{array}$ \\
\hline An5 & $\begin{array}{c}73.03 \% \pm 2.15 \\
\%^{\mathrm{a}}\end{array}$ & $\begin{array}{l}21.02 \% \pm 1.10 \\
\%^{\mathrm{a}}\end{array}$ & $28.8 \% \pm 2.61 \%{ }^{\mathrm{aA}}$ & $6.03 \% \pm 0.23 \%^{\mathrm{a}}$ & $\begin{array}{c}0.68 \% \pm 0.02 \\
\%^{\mathrm{a}}\end{array}$ & $\begin{array}{c}11.26 \% \pm 0.5 \%{ }^{\mathrm{a}} \\
\text { в }\end{array}$ \\
\hline$\Delta M a r p$ & $69.97 \% \pm 6.06 \%{ }^{\mathrm{a}}$ & $12.94 \% \pm 0.29 \% \mathrm{c}$ & $\begin{array}{c}18.56 \% \pm 0.7 \%^{\mathrm{b}} \\
\mathrm{A}\end{array}$ & $\begin{array}{c}69.69 \% \pm 3.03 \\
\%^{\mathrm{c}}\end{array}$ & $\begin{array}{c}0.09 \% \pm 0.00 \\
\%^{\mathrm{b}}\end{array}$ & $\begin{array}{c}0.13 \% \pm 0.005 \\
\%^{\mathrm{bB}}\end{array}$ \\
\hline $\begin{array}{l}\triangle \text { Marp } \\
\text { Comp }\end{array}$ & $83.33 \% \pm 4.41 \%^{\mathrm{b}}$ & $25.65 \% \pm 1.57 \%$ & $30.8 \% \pm 3.25 \%{ }^{\mathrm{aA}}$ & $\begin{array}{c}12.673 \% \pm 0.44 \\
\%^{\mathrm{b}}\end{array}$ & $\begin{array}{c}0.72 \% \pm 0.10 \\
\%^{\mathrm{a}}\end{array}$ & $\begin{array}{c}5.66 \% \pm 1.36 \% \\
\text { в }\end{array}$ \\
\hline $\begin{array}{c}\text { PmvRv36 } \\
71\end{array}$ & $67.783 \% \pm 2.94 \%{ }^{\mathrm{a}}$ & $\begin{array}{c}109.403 \% \pm 4.45 \\
\%^{\mathrm{b}}\end{array}$ & $\begin{array}{c}161.4 \% \pm 11.37 \\
\%^{\mathrm{cA}}\end{array}$ & $\begin{array}{c}5.113 \% \pm 0.48 \\
\%^{\mathrm{a}}\end{array}$ & $\begin{array}{c}1.70 \% \pm 0.27 \\
\%^{\mathrm{c}}\end{array}$ & $\begin{array}{c}33.29 \% \pm 9.2 \%{ }^{\mathrm{c}} \\
\text { в }\end{array}$ \\
\hline
\end{tabular}

Note: The superscript '\#' represents the survival rate under pcit-4.5 divided by survival rates under pcit-6.6, used to correct the effect of pcit buffer, and represents the survival rate under acidic environment. The different superscript ' $\mathrm{a} \sim \mathrm{c}$ ' means the difference in the survival rates within this column is highly significant $(P<0.01)$; meanwhile, the different superscript ' $\mathrm{A} \sim \mathrm{B}$ ' means the difference in the survival rates of the same bacteria between $5^{\text {th }}$ and $14^{\text {th }}$ day is highly significant $(P<0.01)$. Data are means \pm s.d.of triplicate cultures and represent two or more independent experiments.

\subsection{The different growth rates of $\Delta$ Marp, $\Delta$ MarpComp, PmvRv3671, and An5 in Raw264.7}

After 48 hours of infection, most bacteria have been colonized into Raw264.7. The number of bacteria in cells was measured at 48 hours, 96 hours, and 144 hours after infection to calculate the bacterial growth rate. From Figure 7a, few distinct growth characteristics of these four strains could be spotted. From $48 \mathrm{~h}$ to $96 \mathrm{~h}$ after infection, the growth rate in the resting Raw264.7 was significantly faster than in cells after IFN- $\gamma$ stimulation; 4 Marp almost didn't grow in the unstimulated Raw264.7 at 96h-144h. The growth rate of the other three strains between $96 \mathrm{~h}$ to 144 hours was highly significantly faster than in the other periods. Also, between 96 and 144 hours, the four strains were growing significantly faster in the resting Raw264.7 than in stimulated cells. 
Some prominent growth characteristics could be seen in Figure $7 \mathrm{~b}$. The growth rate of $\Delta$ Marp was significantly higher than that of the $\triangle$ MarpComp at 48h-96h after infection in unstimulated Raw264.7. In Raw264.7 after IFN $\gamma$ stimulation, the growth rate of the $\triangle M a r p$ is very significantly slower than that of the other three. The growth rate of PmvRv3671 and An5 are similar in resting Raw264.7 or with IFN $\gamma$ stimulation.

In summary, we found that as time goes on, the growing rates of $\Delta$ MarpComp, PmvRv3671, and An5 all are higher between 96 and 144 h than between 48 and 96 h in resting Raw264.7 cells or Raw264.7 cells with IFN $\gamma$ stimulation, but the situation of $\Delta$ Marp is contrasting. This indicated that the presence or absence of the Rv3671c gene made a notable influence on the growth rate of $M$. bovis in Raw264.7 cells.
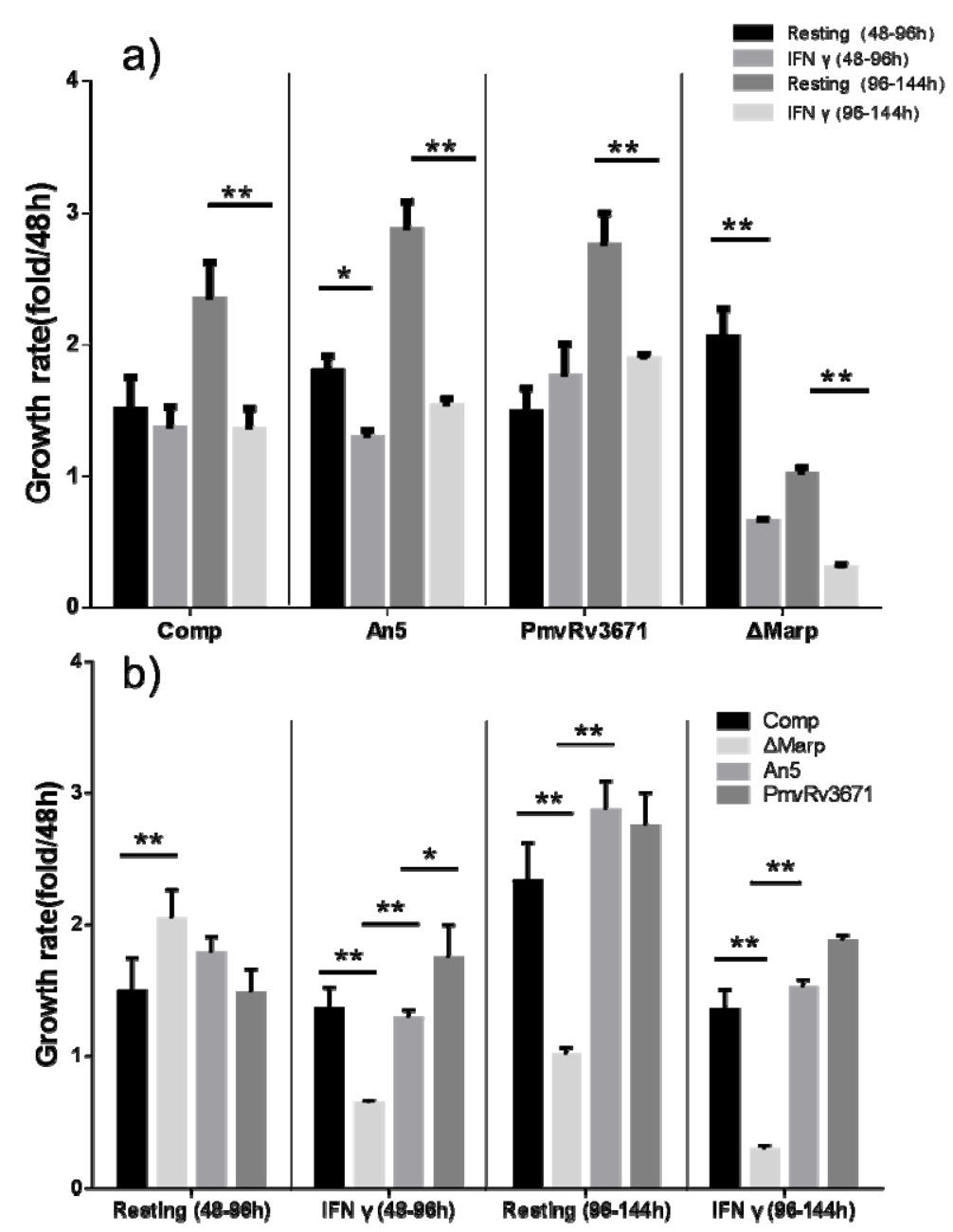

Figure 7. Growth rates of M. bovis An5 , $\Delta$ Marp, $\Delta$ MarpComp, and PmvRv3671 respectively in resting Raw264.7 and Raw264.7 stimulated by IFN $\gamma$. a) Growth rates of these four strains in resting Raw264.7 and Raw264.7 stimulated by IFN $\gamma$ in different periods; b)Comparison of the growth rates of these four strains in resting Raw264.7 and Raw264.7 stimulated by IFN $\gamma$. '*' or'**' means the differences of the growth rates between the two groups is significant $(p<0.05)$ or highly significant $(p<0.01)$. Data are means \pm s.d.of triplicate cultures and represent two or three independent experiments. In some panels, error bars are too small to be seen. 


\section{Discussion}

Mycobacterium tuberculosis is a typical intracellular pathogen, which can survive in extreme conditions such as hypoxia, nitric oxide killing, and nutritional deficiencies in macrophages, and suppress the host immune response through multiple escape mechanisms to avoid clearance by the body (Mena Cimino et al., 2012; Naffin-Olivos et al., 2014; Philips and Ernst, 2012; Walburger et al., 2004). Although some are killed under those changing environmental conditions, the escaped MTB could still mainly parasitize in macrophages and replicate or replicate into a stage of dormancy rendering itself extremely resistant to host defence (Mena Cimino et al., 2012). In a word, the pathogenic mechanisms in hosts are very complicated and the $R v 3671 c$ gene is essential for the process of acid resistance. M. bovis is very similar to MTB but has a wider host, and it has been confirmed that this gene also presented in $M$. bovis with our previous experiments, but whether there are other biological functions like growth involved remains to be a mystery to be explored. To further verify the functions and biological characteristics of the gene $R v 3671 c$ in $M$. bovis, the Rv3671c knockout strain was prepared using the method as previously (Jain et al., 2014), and the shuttle vector was utilized to construct the complemented strain and overexpression strains.

According to the measurement results of the growth curves of these four strains in pH6.6 7H9, the $\Delta$ Marp could grow normally, which is similar to the results reported previously (Botella et al., 2017), but the CFU of PmvRv3671 on $14^{\text {th }}$ day is more than five times with the CFU of the $\Delta$ Marp. This suggested that the deletion of Rv3671c in M. bovis could affect its growth, but it is not an essential gene for $M$. bovis growing in standard $7 \mathrm{H} 9$ medium. However, when the experiment overexpressed $R v 3671 c$ gene in M. bovis, PmvRv3671 showed a stronger growth performance in a nutritious medium, indicating that the gene might enhance the related metabolisms of M. bovis. Although the $\mathrm{OD}_{600}$ value of PmvRv3671 on the $14^{\text {th }}$ day was lower than that of the An5 strain, it might be related to the change in color of the bacteria, and this characteristic could be observed in PmvRv3671.

From the growth curve measurement results of the four strains cultured in pH6.6 7H9, it was found that the growth results of PmvRv3671 also outperformed An5 and this point was amplified in acidic nutrient medium (pH4.5-7H9). Meanwhile, $\Delta$ Marp could not maintain the growing ability in that acidic medium. The above two points both indicated that the Rv3671c gene infected the growth of M. bovis, especially in an acidic nutrient medium. Vandal (Vandal et al., 2008) previously performed another experiment on the Mtb H37Rv at 7H9-Tw-4.5 medium, which turned out to be that the inoculated Mtb was almost completely killed on the 15th day. After that, they proposed Tween-80 might release free fatty acids to kill Mtb, so Tween- 80 was replaced by nonhydrolyzable tyloxapol as the dispersing agent, but the change still failed to observe the growth of Mtb in pH4.5 7H9. This exhibited that the wild-type $M$. bovis An5 share similar acid-resistance mechanisms like Mtb, because the M. bovis An5 cannot grow in pH4.5 7H9 either. In addition, PmvRv3671 grew better in acidic environment (pH5.0 $7 \mathrm{H} 9$ ) than the neutral environment, which suggested that $R v 3671 c$ gene plays a more important role in acidic environments; it might be due to the acidic environment trigger more pathways by activating Rv3671c gene and its related downstream, like ripA response for cell elongation and chain formation (Botella et al., 2017); Flannagan also believes that acidic conditions will activate the degradation of bacterial lipids and proteins (Flannagan et al., 2009). For instance, Escherichia coli (E. coli) could use amino acid decarboxylase/antiporter systems that utilize glutamate, arginine, and lysine as substrates, to increase its cytoplasmic pH (Foster, 2004), and Marp, a serine protease, might work in a similar way to help the growth of mycobacteria.

By observing the survival rate results of the four bacteria in $\mathrm{pH} 4.5$ and $\mathrm{pH} 6.6$ maintenance solutions (pcit buffer), it can be seen that the rate of $M$. bovis in neutral and acidic environments both decrease as time goes on, except $\triangle$ Marp in neutral environment. PmvRv3671 presented the lowest survival rates on both the $5^{\text {th }}$ and the $14^{\text {th }}$ day when culturing in the pcit $\mathrm{pH} 6.6$ environment, but no significant difference with An5 was observed. Notably, 4 Marp was extremely stable in the pcit pH 6.6 environment, and even on the 14 th day, the survival rate was the same as the $5^{\text {th }}$ day $(69.97 \%)$. This indicates that the Rv3671c gene does not promote the survival of $M$. bovis in the neutral maintenance environment; contrarily, $\triangle$ Marp can survive longer in a neutral environment and it might since the deletion of the Rv3671c gene which could slow parts of the metabolisms. Previously it has been proved that Marp cleaves the peptidoglycan hydrolase RipA to activate RipA in acid environment (Botella et al., 2017). When Marp was knockout in $\Delta$ Marp, the failure of RipA processing leads to cell 
extension and chain formation, which is a sign that the separation of progeny bacteria stops. The lack of interaction with RipA in $\triangle M a r p$ resulted in the activity of RipA is greatly reduced. This inhibited the bacterial growth rate, which is why it could be observed that PmvRv3671 grows faster than An5 and An5 also grow faster than $\Delta$ Marp. This also explains why the deleted strain in the neutral maintenance solution could be so stable even on the $14^{\text {th }}$ day. Surprisingly, the proteomic comparison exhibited that the ATP $\gamma$ chain in the deleted strain was significantly more than that in the wild strain (Small, 2013). It further suggests that the $\triangle$ Marp use and consume ATP less efficiently and the deletion of Marp in $M$. bovis might slow down its energy metabolisms.

On the other hand, the survival rate of PmvRv3671 in $\mathrm{pH} 4.5$ maintenance buffers is even higher than that in $\mathrm{pH} 6.6$ maintenance buffer, providing a similar outcome to the results culturing it in $7 \mathrm{H} 9$ medium. Comparing the corrected survival rates, it can be found that after removing the influence of the maintenance solution, the acid resistance of them on the $14^{\text {th }}$ day all were significantly decreased than that on the $5^{\text {th }}$ day. Apparently, the $\Delta$ Marp still remain part of acid resistance; even without Marp there are still other acid-resistance mechanisms like the predicted magnesium transporter MgtC (Buchmeier et al., 2000); outer membrane protein OmpATB (Song et al., 2011); a two-component regulator PhoPR (Rohde et al., 2007); a gene locus regulated by PhoPR in response to acid aprABC (Abramovitch et al., 2011) in mycobacteria. Whatever, the comprehensive acid resistance of M. bovis still becomes weaker in acid maintenance solution as time goes on.

According to the growth rate of these four strains in Raw264.7 macrophages, it can be stated that mature macrophages presented a stronger killing capacity on mycobacteria. The growth rates of $M$. bovis An5 , $\Delta$ MarpComp, and PmvRv3671 all are greater than 1.0 in mature macrophages, which indicates that these three strains can grow or even further infected macrophages; 1 Marp, however, is dying and cannot maintain the infection state for a long time in mature macrophages, even in the resting macrophage in the $96-144 \mathrm{~h}$. Therefore, it can be seen that the deletion of the $R v 3671 c$ gene prevents $M$. bovis from forming a long-term infection in mature macrophages, indicating that the Rv3671c gene is essential for the survival of $M$. bovis in mature macrophages like previous in MTB (Vandal et al., 2008). In addition, PmvRv3671 exhibited a faster growth rate than wild-type strains in mature macrophages like it did in $7 \mathrm{H} 9$ at $\mathrm{pH}$ 5.0. Besides, comparing their growth rates in macrophage with IFN- $\gamma$ stimulated, it proved that mature macrophage can not eliminate all intracellular bacteria. However, comparing their growth in unstimulated Raw264.7 cells, even if mature macrophages can not eliminate all intracellular bacteria, they remain the inhibitory ability to intracellular mycobacteria.

Tuberculosis is an important infectious disease with large number of deaths caused by a single pathogen in the world and is also considered the leading cause of death after AIDS (WHO, 2019).

The main pathogenic bacteria of tuberculosis are Mycobacterium tuberculosis (Mtb) and Mycobacterium bovis (M. bovis). Mechnikov indicated that macrophages would kill most of the ingested microorganisms by acidifying its interior, but Mtb could use its waxy cell wall to resist this acidification (Mechnikov, 1988). According to previous reports, when Bacille Calmette-Guérin (BCG), an attenuated variant of $M$. bovis, was exposed to $\mathrm{pH} 5.5$ in vitro, it ceased replicating and Mtb continued to divide slowly unless the $\mathrm{pH}$ was lowered to 4.5 . With the addition of $0.5 \mathrm{mM}$ nitrite to 7H9 medium at pH 5.5, Mtb would also stop replicating. However, it is in a balanced state, where the Mtb could survive for several days, and the bacterial can return to a normal growth rate after replacing the medium with standard 7H9 (Bryk et al., 2008; Gold et al., 2012; Vandal et al., 2008).

Based on that, Vandal used the acidic environment to screen 10,100 MTB transposon mutants and found a mutant that cannot maintain its intracellular $\mathrm{pH}$ homeostasis under a $\mathrm{pH} 4.5$ environment (Vandal et al., 2008). Because the gene Rv3671c product played an essential role in the acid resistance of $\mathrm{Mtb}$, it is named as Mycobacterial acid resistance protease (Marp). Marp was identified as a transmembrane serine peptidase with a protease domain located in the periplasm, as confirmed by a prudent analysis of its mutations, homology modeling, crystallography, and substrate profiling (Biswas et al., 2010; Small et al., 2013; Vandal et al., 2008).

In summary, culturing the four bacteria grew in $7 \mathrm{H} 9$ medium at $\mathrm{pH} 5.0$ or $\mathrm{pH} 6.6$ and maintenance buffer at $\mathrm{pH} 4.5$, the lived number of over-expressing strain PmvRv3671 is significantly more than wild-type strain M.bovis, $\Delta$ MarpComp and $\Delta$ Marp on the $14^{\text {th }}$ day. After removing the effects of citrate solution, it can be found that the acid resistance abilities of them all are significantly lower on the $14^{\text {th }}$ day than that on the $5^{\text {th }}$ day. Using them infected Raw264.7 macrophages with IFN $\gamma$ stimulation, the growth rate of the PmvRv3671 is faster than An5, $\Delta$ Marp and $\Delta$ MarpComp. In conclusion, the gene Rv3671c in $M$. bovis is not only related to acid resistance but also affected the growth of M. bovis, even 
bioRxiv preprint doi: https://doi.org/10.1101/2020.09.15.298273; this version posted September 15, 2020. The copyright holder for this preprint (which was not certified by peer review) is the author/funder. All rights reserved. No reuse allowed without permission.

the metabolisms of energy. 


\section{References}

Abramovitch, R.B., Rohde, K.H., Hsu, F.F., Russell, D.G., 2011. aprABC: a Mycobacterium tuberculosis complex-specific locus that modulates $\mathrm{pH}$-driven adaptation to the macrophage phagosome. Mol Microbiol 80, 678-694.

Botella, H., Vaubourgeix, J., Lee, M.H., Song, N., Xu, W., Makinoshima, H., Glickman, M.S., Ehrt, S., 2017a. Mycobacterium tuberculosis protease MarP activates a peptidoglycan hydrolase during acid stress. The EMBO journal 36, 536-548.

Bardarov, S., Bardarov, S., Pavelka, M.S., Sambandamurthy, V., Larsen, M., Tufariello, J., Chan, J., Hatfull, G., Jacobs, W.R., 2002. Specialized transduction: an efficient method for generating marked and unmarked targeted gene disruptions in Mycobacterium tuberculosis, M. bovis BCG and M. smegmatis. Microbiology 148, 3007-3017.

Biswas, T., Small, J., Vandal, O., Odaira, T., Deng, H., Ehrt, S., Tsodikov, O.V., 2010. Structural insight into serine protease $R v 3671 c \mathrm{c}$ that Protects $M$. tuberculosis from oxidative and acidic stress. Structure 18, 1353-1363.

Bryk, R., Gold, B., Venugopal, A., Singh, J., Samy, R., Pupek, K., Cao, H., Popescu, C., Gurney, M., Hotha, S., Cherian, J., Rhee, K., Ly, L., Converse, P.J., Ehrt, S., Vandal, O., Jiang, X., Schneider, J., Lin, G., Nathan, C., 2008. Selective killing of nonreplicating mycobacteria. Cell Host Microbe 3, 137-145.

Buchmeier, N., Blanc-Potard, A., Ehrt, S., Piddington, D., Riley, L., Groisman, E.A., 2000. A parallel intraphagosomal survival strategy shared by mycobacterium tuberculosis and Salmonella enterica. Mol Microbiol 35, 1375-1382.

Ehrt, S., Schnappinger, D., 2009. Mycobacterial survival strategies in the phagosome: defence against host stresses. Cell Microbiol 11, 1170-1178.

Flannagan, R.S., Cosio, G., Grinstein, S., 2009. Antimicrobial mechanisms of phagocytes and bacterial evasion strategies. Nat Rev Microbiol 7, 355-366.

Foster, J.W., 2004. Escherichia coli acid resistance: tales of an amateur acidophile. Nat Rev Microbiol 2, 898-907.

Gao, X., Guo, X., Li, M., Jia, H., Lin, W., Fang, L., Jiang, Y., Zhu, H., Zhang, Z., Ding, J., Xin, T., 2019. Interleukin 8 and Pentaxin (C-Reactive Protein) as Potential New Biomarkers of Bovine Tuberculosis. J Clin Microbiol 57.

Gold, B., Pingle, M., Brickner, S.J., Shah, N., Roberts, J., Rundell, M., Bracken, W.C., Warrier, T., Somersan, S., Venugopal, A., Darby, C., Jiang, X., Warren, J.D., Fernandez, J., Ouerfelli, O., Nuermberger, E.L., Cunningham-Bussel, A., Rath, P., Chidawanyika, T., Deng, H., Realubit, R., Glickman, J.F., Nathan, C.F., 2012. Nonsteroidal anti-inflammatory drug sensitizes Mycobacterium tuberculosis to endogenous and exogenous antimicrobials. Proc Natl Acad Sci U S A 109, 16004-16011.

Jain, P., Hsu, T., Arai, M., Biermann, K., Thaler, D.S., Nguyen, A., González, P.A., Tufariello, J.M., Kriakov, J., Chen, B., 2014. Specialized transduction designed for precise high-throughput unmarked deletions in Mycobacterium tuberculosis. MBio 5, e01245-01214.

Mena Cimino, C.T., Namouchi, A., Dubrac, S., Gicquel, B., Gopaul, D.N., 2012. Identification of DNA binding motifs of the Mycobacterium tuberculosis PhoP/PhoR two-component signal transduction system. PloS one 7.

Mechnikov, II, 1988. Immunity in infective diseases. By Il'ia Il'ich Mechnikov, 1905. Rev Infect Dis $10,223-227$.

Naffin-Olivos, J.L., Georgieva, M., Goldfarb, N., Madan-Lala, R., Dong, L., Bizzell, E., Valinetz, E., Brandt, G.S., Yu, S., Shabashvili, D.E., 2014. Mycobacterium tuberculosis Hip1 modulates macrophage responses through proteolysis of GroEL2. PLoS pathogens 10.

Van Kessel, J.C., Hatfull, G.F., 2007. Recombineering in Mycobacterium tuberculosis. Nat Methods 4, $147-152$.

Vandal, O.H., Pierini, L.M., Schnappinger, D., Nathan, C.F., Ehrt, S., 2008. A membrane protein preserves intrabacterial $\mathrm{pH}$ in intraphagosomal Mycobacterium tuberculosis. Nat Med 14, 849-854.

Xin, T., Gao, X., Yang, H., Li, P., Liang, Q., Hou, S., Sui, X., Guo, X., Yuan, W., Zhu, H., Ding, J., Jia, H., 2018. Limitations of Using IL-17A and IFN-gamma-Induced Protein 10 to Detect Bovine Tuberculosis. Front Vet Sci 5, 28. 
World Health Organization. Global tuberculosis Report. Geneva :WHO, 2019.

Pesciaroli, M., Alvarez, J., Boniotti, M.B., Cagiola, M., Di Marco, V., Marianelli, C., Pacciarini, M., Pasquali, P., 2014. Tuberculosis in domestic animal species. Res Vet Sci 97 Suppl, S78-85).

Philips, J.A., Ernst, J.D., 2012. Tuberculosis pathogenesis and immunity. Annual Review of Pathology: Mechanisms of Disease 7, 353-384.

Rohde, K.H., Abramovitch, R.B., Russell, D.G., 2007. Mycobacterium tuberculosis invasion of macrophages: linking bacterial gene expression to environmental cues. Cell Host Microbe 2, 352-364.

Small, J.L., O'Donoghue, A.J., Boritsch, E.C., Tsodikov, O.V., Knudsen, G.M., Vandal, O., Craik, C.S., Ehrt, S., 2013. Substrate specificity of MarP, a periplasmic protease required for resistance to acid and oxidative stress in Mycobacterium tuberculosis. J Biol Chem 288, 12489-12499.

Vandal, O.H., Nathan, C.F., Ehrt, S., 2009. Acid resistance in Mycobacterium tuberculosis. J Bacteriol 191, 4714-4721.

Vandal, O.H., Pierini, L.M., Schnappinger, D., Nathan, C.F., Ehrt, S., 2008. A membrane protein preserves intrabacterial $\mathrm{pH}$ in intraphagosomal Mycobacterium tuberculosis. Nat Med 14, 849-854.

Snapper, S.B., Melton, R.E., Mustafa, S., Kieser, T., Jacobs, W.R., Jr., 1990. Isolation and characterization of efficient plasmid transformation mutants of Mycobacterium smegmatis. Mol Microbiol 4, 1911-1919.

Small, J.L., 2013. Mechanisms of pH homeostasis and cytochrome C maturation in Mycobacterium tuberculosis. Weill Medical College of Cornell University.

Song, H., Huff, J., Janik, K., Walter, K., Keller, C., Ehlers, S., Bossmann, S.H., Niederweis, M., 2011. Expression of the ompATb operon accelerates ammonia secretion and adaptation of Mycobacterium tuberculosis to acidic environments. Mol Microbiol 80, 900-918.

Walburger, A., Koul, A., Ferrari, G., Nguyen, L., Prescianotto-Baschong, C., Huygen, K., Klebl, B., Thompson, C., Bacher, G., Pieters, J., 2004. Protein kinase G from pathogenic mycobacteria promotes survival within macrophages. Science 304, 1800-1804.

Weiss, G., Schaible, U.E., 2015. Macrophage defense mechanisms against intracellular bacteria. Immunol Rev 264, 182-203.Ansari, I.H., Kwon, B., Osorio, F.A., Pattnaik, A.K., 2006. Influence of N-linked glycosylation of porcine reproductive and respiratory syndrome virus GP5 on virus infectivity, antigenicity, and ability to induce neutralizing antibodies. J. Virol. 80, 3994-4004. 